\title{
Vehicle Tracking/Feedback System Using Gps Technology
}

\author{
Mbaocha C. Christian \\ Department of Electrical/Electronic Engineering \\ Federal University of Technology Owerri, Imo State, Nigeria
}

\begin{abstract}
Today almost everywhere car theft abounds and for this reason it is necessary to know at any time where ones vehicle is located and in what condition. This work aimed at designing a navigation/tracking system using a parallax GPS module, PIC16F873 microcontroller, PL-2303 USB microcontroller and MAX232 converter. Sony Ericsson T69 and a laptop with a visual basic programmed interface aids in giving output information for the tracking and navigation sub systems respectively. The GPS receiver interacts with the satellite in space and receives data i.e. location information in the form of radio waves. This information is sent to the USB microcontroller and the 8-bit microcontroller as raw NMEA 0183 string in TTL level. The 8-bit microcontroller stores the information in memory and when requested is sent through an RS-232 converter that converts the TTL message to RS level message that the GSM modem can understand. The GSM modem then sends the strings as a text to an IMEI number already programmed on the microcontroller. The location as at the time of transmission is shown on the graphic user interface where the message is keyed into.
\end{abstract}

Key words: Navigation system, Tracking, Global Positioning System (GPS), Converter, Graphic User Interface (GUI)

\section{Introduction}

Mankind has always reacted to problems with strong determination aimed at providing better solutions to difficulties. From the use of celestial navigation to the use of compass man has always searched for a means of accurately locating his position on the globe with precision and accuracy necessary to avoid tragedy and to reach his intended destination. One could also want to locate a specific place in a locality familiar to him. This could be a shopping mall, a petrol station, a hotel, or even an office.

The Global Positioning System (GPS), is a popular satellite navigation system which makes use of a constellation of more than two dozen GPS satellites to transmit precise radio wave signals in any weather, day or night, anywhere on Earth. This allows a GPS receiver in an unobstructed line of view to synchronize with the satellite to determine its current horizontal location to the nearest 15 meters radius in latitude, longitude and altitude by connecting to just four satellites in its horizon. It provides accurate three-dimensional position (latitude, longitude, and altitude), velocity and precise time traceable to Coordinated Universal Time (UTC). To make the device user friendly, the information from the GPS is translated to information that can be understood via a map [1].

The aim of this work is to integrate readily available materials with the implementation of the knowledge of software engineering and basic electrical and electronics to build an easy to use, computerized navigation/tracking system capable of tracking the movements of the target onboard or remotely as well as locating areas of interest within a given locality.

This device can be used by surveyors to ease the problems of marking virgin terrains, and producing Maps of oil fields. The tourism potential of an area can be increased three folds by the application of this device. Because of the tracking capabilities of the device it can easily be used to monitor the movements of a vehicle as well as for vehicle recovery [2].

\section{Design Objectives}

This device is required to have a speed as high as possible. It should be a dual purpose device that can track a position as well as find the direction to a specific location.

The system has a navigation update rate of atmost once every two seconds, a velocity accuracy of around $+/-0.2$ meter per second and a position accuracy not greater than $+/-5$ meters.

III. Block Diagram Description

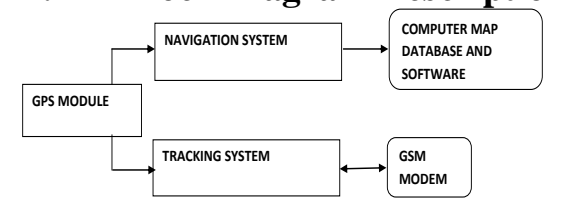

Fig. 1. Block Diagram of the hardware components of navigation/tracking device. 
Fig1. represents the block diagram of the system. The GPS receiver interacts with the satellite in space and receives data i.e. location information in the form of radio waves. This information is sent to the input of both the navigation system and the tracking system. The 8-bit microcontrollers in both systems store the information in memory and later sent through an RS-232 converter that converts the TTL message to RS level message that the GSM modem (used as feedback system) can understand. The GSM modem then sends the strings as a text to an IMEI number already programmed on the microcontroller. The location as at the time of transmission is shown on the graphic user interface where the message is keyed into.

Meanwhile, the USB microcontroller for the navigation system sends the information to a computer system through a data cable where the software reads the port number and initializes the navigation system written on it.

\subsection{GPS Receiver}

This GPS receiver was designed by a cooperation of GRAND IDEA STUDIO (www.grandideastudio.com) and PARALLAX INC. to use this receiver module because it offers GPS solutions even in very short distances (1.92" long and 1.24" wide). The electronic connection of the parallel GPS receiver circuit is shown in Fig.2 [3].

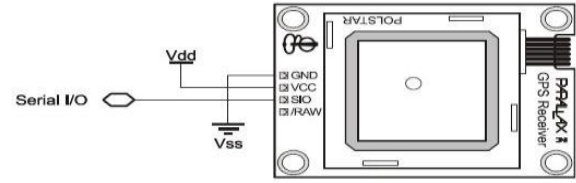

Fig 2: Parallax Gps Receiver Showing The Electronic Connections

Upon power up of the GPS Receiver Module in a new location, the Module may take up to five minutes or more to acquire a fix on the necessary minimum number of four satellites. During this time, the red LED on the Module will blink. When enough satellites are acquired for the Module to function properly, the red LED will remain solid red. Due to a variety of conditions, the number of satellites may vary at any given time.

\subsection{The Tracking System}

The project requires a suitable microcontroller to co-ordinate all activities. This includes reading data sent by the GPS receiver module and communicating this data to the GSM modem. This ideal controller should be able to handle these operations in terms of memory and processing speed. In this work, the PIC16F876A microcontroller is used because of it has large memory and suitable processing speed [4]. Using 78L05 a regulated power supply is provided to the microcontroller chip and its clocking signal is set by $4 \mathrm{MHz}$ crystal quartz. Most GSM devices provide input and output using normal logic levels while others provide RS232 levels. The RS232 converter used in this project is MAX232 microchip [5].

The Navigation Subsystem contains the USB controller PL-2303 that interfaces and transfers all data from all other parts of the system to the USB port of the computer.

\section{Software Design Flowchart And Description}

Visual Basic is an event driven programming language. A program is developed by working on an interface. This includes setting up controls, writing codes behind them so that when users decide to use them, they click on the controls and the code runs and carries out a specific task. Visual Basic can also be described as a Rapid Application Development (RAD) tool. This is because it is very easy or fast to use. A Visual Basic program can be developed in a matter of minutes or hours as the case may be.

The main objective of the software design to this project is to create a graphic user interface (GUI) that would enable the user of the navigation device to see his position at every point in time. This implies that the GUI enables us to see what happens within the GPS module and USB microcontroller in a manner we can easily understand

\subsection{Google Earth}

Google earth is a virtual globe, map and geographic information, originally called Earth Viewer created by Keyhole, Inc. It maps the Earth by the superimposition of images obtained from imagery, aerial photography and GIS globe. In this project, Google Earth was used to get the map of FUTO which is used as a case study. The map gotten covers from FUTO front gate through senate and major buildings up school such as SEET HEAD, SOHT, SMAT, SAAT, SOSC and SOSC extension, SLT, workshops 2 and 3, main and pilot library, 750 and 500 capacity lecture theatres, registry and finally hostels A, B, C, D, and PG hostels. The map is shown on Appendix A.

\subsection{Visual Basic}

The GUI of this project is a program developed with VB .NET version 2005 (Visual Basic 8.0). It has some functions intended to reinforce Visual Basic .NET's focus as a rapid application development platform and further differentiate it from C++. Once the USB cable is plugged into the system through the serial port, port initialization takes place [6]. The computer automatically attaches the USB to a particular port number. When the port is ready, data is read from the cable. But the program was written in such a manner that if port

$$
\text { www.iosrjournals.org }
$$


initialization is not complete, no further action is taken while port initialization takes place again. This continues until the port is certified ready. Next is reading of data from the microcontroller after which the data is matched with already prescribed data. This leads to the result being displayed on the map in the form of a red dot on the exact location corresponding to the already read and matched data. After this, the sequence stops automatically until the user moves from a point to another. This movement automatically leads to the GPS acquiring fresh location data and sends it to the computer through the USB cable and the cycle repeats itself [7]. It should be noted that data sent to the microcontroller is done at every instant the GPS receives a fresh location data which is as a result of movement from one point to the other. This procedure is pictorially explained in the flowchart Fig3.

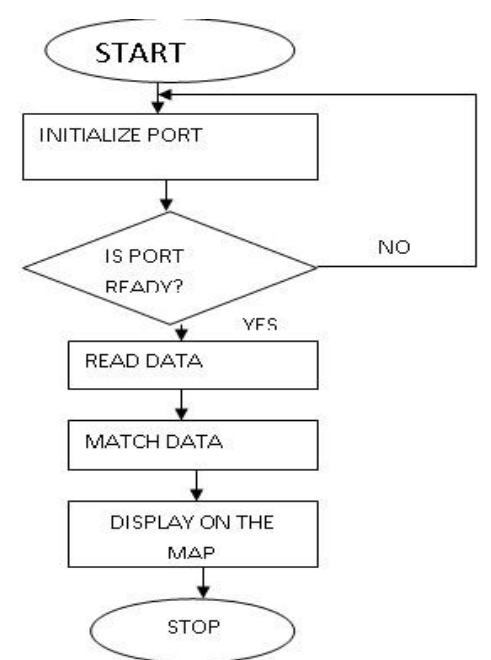

Fig 3: Visual Basic Implementation Flowchart

\subsection{Programming Microcontroller}

The flowchart in fig4 illustrates the operation of microcontroller in the implemented system. When the system starts, the ports are initialized. At the receipt of a signal, the controller confirms if it is low or high. If it is high, it clears all data from its memory and request position data from the GPS. Once the data is received, it is stored and sent to the phone [8].

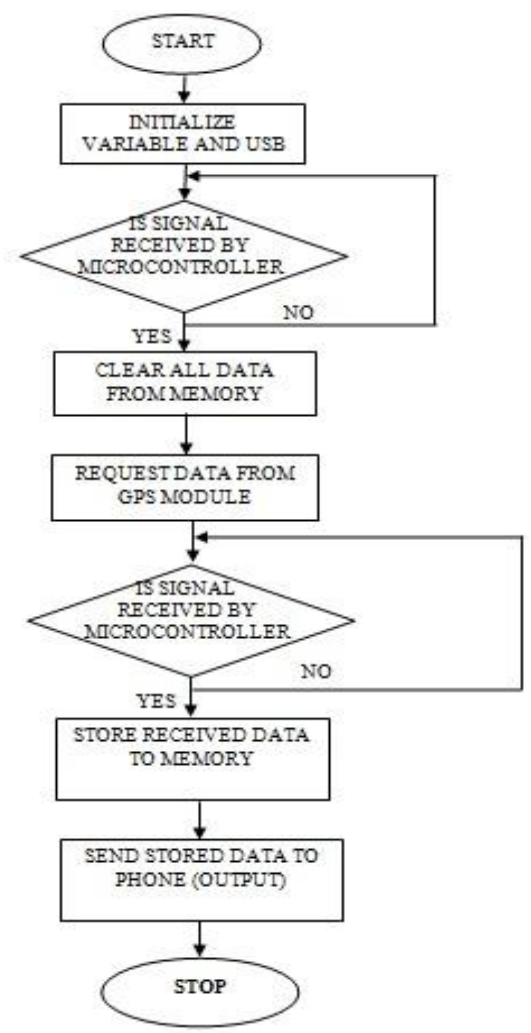

Fig 4: Microcontroller Programming Flowchart 


\section{Complete Circuit Design}

The designed system has two major parts, the tracking subsystem and the navigation subsystem. These are shown in fig 5 and 6.

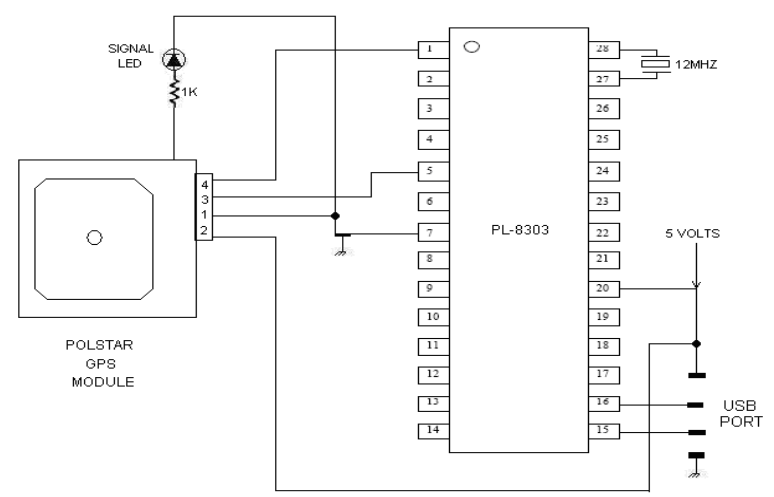

Fig 5 Circuit Of Navigation Subsystem

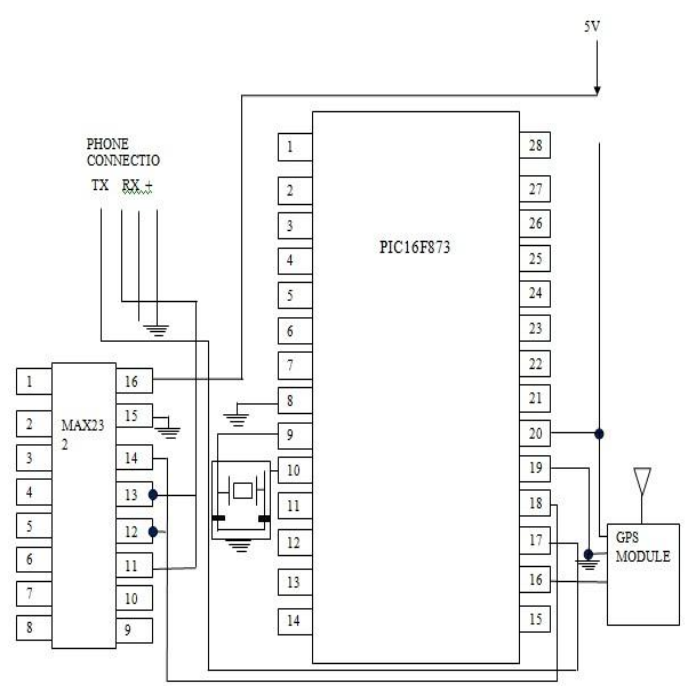

Fig 6 The Tracking Subsystem

\section{Simulation and Testing}

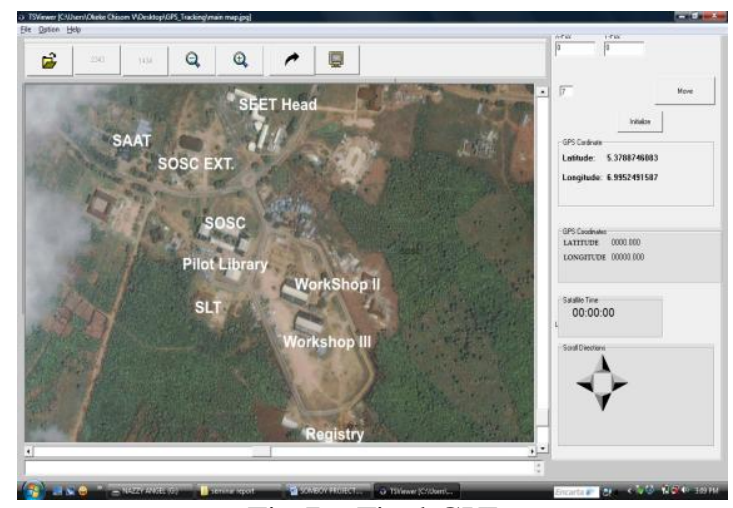

Fig 7: Final GUI

The device was tested in the streets of Owerri with taxi drivers as part of post implementation testing. The whole device with a laptop was placed on the passenger seat of the car. A map of Owerri town resolved to Okigwe road was integrated into the graphic user interface and the movement of the cab tracked (see fig 7). The device worked well but for the complication in designing and constructing the system.

The real-time behavior of the microcontroller was simulated before implementation using PIC simulator IDE. This was done by running the call-to-security service program in the simulation environment. Also, debugging was carried out using the simulator. A representation of the simulation environment is shown in the fig 8 . 


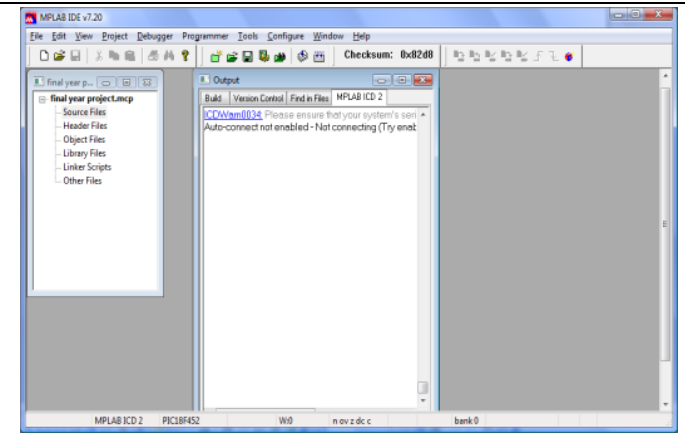

Fig 8 MP-Lab Simulator

This simulation is highly necessary because verification of the written instructions must be carried out before being uploaded into the program memory of the microcontroller. These instructions are uploaded using the PIC programmer [9].

\subsection{GPS Modem Testing}

The GPS modem is latched to a system through a serial or USB port and switched on. The data strings are seen on the hyper terminal screen. The GPS receiver has its format in NMEA 0183 format. The format was compared to precise location information on the internet to ascertain that the receiver is accurate. Below is an example of NMEA0183 GPS strings as seen from a hyper terminal. \$GPGGA, 170834,4124.8963,N,08151.6838,W,1,05,1.5,280.2,M,-34.0,M,,,*75

\$GPGSA,A,3,19,28,14,18,27,22,31,39,,,,,1.7,1.0,1.3*34

\$GPGSV,3,2,11,14,25,170,00,16,57,208,39,18,67,296,40,19,40,246,00*74

\$GPRMC,220516,A,5133.82,N,00042.24,W,173.8,231.8,130694,004.2,W*70

After all the testing was done and the best components assembled, the whole team went forward to solder on the components to the vero board using the best techniques and safety measures. The circuit design was strictly adhered to.

\section{Conclusion}

The Global Positioning System (GPS), is a popular satellite navigation system which makes use of a constellation of more than two dozen GPS satellites to transmit precise radio wave signals in any weather, day or night, anywhere on Earth. This allows a GPS receiver in an unobstructed line of view to synchronize with the satellite to determine its current horizontal location to the nearest 15 meters radius in latitude, longitude and altitude by connecting to just four satellites in its horizon. It provides accurate three-dimensional position (latitude, longitude, and altitude), velocity and precise time traceable to Coordinated Universal Time (UTC). To make the device user friendly, the information from the GPS is translated to information that can be understood via a map.

The aim of this work is to integrate readily available materials with the implementation of the knowledge of software engineering and basic electrical and electronics to build an easy to use, computerized navigation/tracking system capable of tracking the movements of the target onboard or remotely as well as locating areas of interest within a given locality.

This project can be used by surveyors to ease the problems of marking virgin terrains, and producing Maps of oil fields. The tourism potential of an area can be increased three folds by the application of this device. Because of the tracking capabilities of the device it can easily be used to monitor the movements of a vehicle as well as for vehicle recovery.

\section{REFERENCES}

1. "GPS user module guide" http//www.axiomnav.com (20/09/08)

2. "Introduction to GSM/GPRS wireless Modems" http://www.developers Home.com (11/09/08)

3. H.K. Morgan, Tracking systems worldwide ( $3^{\text {rd }}$ edition Keith nelson printers) 2005.

4. M. Nebojsa, Microcontrollers for beginners (Microchip technology inc USA) 2003

5. PIC16F87X Data Sheet. http://www.microchip.com (12/10/08)

6. S.R. Pressman, Software Engineering; A Practioners' Approach,(2 ${ }^{\text {nd }}$ edition, McGraw hill ) 1987.

7. J.P. Pratt and J.J Adamski, Database management systems and design ( $2^{\text {nd }}$ edition Boyd and Fraser publishing company) 2002.

8. B. Reselman and W. Pruchiak, Visual basic Tutor $\left(1^{\text {st }}\right.$ edition Macmillian computer Publishing) 2000

9. M.G. Say, Electrical Engineer's reference book, $15^{\text {th }}$ edition, 1993 